which can also be written, using the homology relation between $R$ and $X$, in the form :

$$
\frac{R}{M}>\frac{1}{n+1 \cdot 75} \frac{G m_{0}}{Q X_{1}}
$$

Remembering that $M \sim L^{1 / 3}$ and $R \sim L^{1 / 2} T_{\text {eff. }}{ }^{-2}$, we can write our condition for the critical line in the form : $L \sim T_{\text {eff. }}{ }^{12}$, which roughly coincides with the equation of the line of pulsating variables. Moreover, substituting the numerical values, we find that the line given by this condition really passes through the location of these stars in the diagram. Thus the line of the pulsating stars represents the geometrical locus of points where the gravitational contraction begins to play a more important part than the nuclear energy production. Above this line no 'lateral' structure can be expected, and any star goes through this region rapidly contracting and radiating the gravitational energy liberated in such contraction. This process stops, however, as soon as the star reaches the main sequence where, due to a very high abundance of hydrogen, the energy liberation through the catalysing action of carbon and nitrogen becomes again the predominant process.

Let us now imagine a star which has just passed this limiting line and the source of energy of which is therefore mostly gravitational. If, for some reason, the radius of the star decreases slightly, the central temperature will increase correspondingly and the nuclear energy production will become of importance, giving to the star an impulse to expand. But as soon as the expansion starts and the central temperature again drops, the nuclear energy production becomes again negligible as compared with gravitation. It is easy to see that this effect will cause an undamped pulsation-in which the star will be, so to speak, 'bouncing on the critical line'. The pulsation will last as long as there is still sufficient amount of the reacting element, and there is every reason to believe that the observed pulsations of stars near the limiting line are due to such a process. After the amount of the element responsible for the pulsation becomes quite negligible, the star will rapidly pass the region of gravitational contraction and will become a star of the upper part of the main sequence.

(4). It remains now only to consider the later stages of stellar evolution, when the star, after staying sufficiently long in the main sequence, has consumed all its original content of hydrogen and enters into the stage of the final gravitational con. traction. It is known ${ }^{11}$ that the final stage of such contraction depends essentially on the mass of the star.

If the mass of the star is smaller than the limiting value $1.4 M \odot$ (for stars completely deprived of hydrogen), a degenerated electron gas will begin to be formed at a certain stage of contraction, and the star will tend to the state of very high density and low luminosity characteristic of the stars known at present as white dwarfs.

For stars of larger mass, no lower limit of contraction exists, because the gravitational pressure of the outer layers cannot be balanced at all by the pressure of the degenerated electron gas. At a certain stage of the contraction one may expect that in the interior of the star a large amount of free neutrons will begin to be formed, leading to a rapid collapse of the whole star and to the liberation of tremendous amounts of gravitational energy ${ }^{12}$.

The question as to how such explosions can be connected with the observed phenomena of novce and supernovce is not yet quite clear, and we will not enter here into its discussion.

Summing up, we can say that, due to the application of our present knowledge of nuclear physics, the problem of stellar energy sources and the main features of stellar evolution can be considered at present as practically solved.

\footnotetext{
- Gamow, G., and Teller, E., Phys. Rec., 55, 791 (1939).

"Chandrasekhar, S., "Stellar Structure" (Chicago Univ. Press, 1939). 10 Gamow, G., Phys. Rev., 55, 790 (1939).

"Chandrasekhar, S., Mon. Not. Roy. Ast. Soc., 91, 456 (1931) and following publications.

12 Baade, W., and Zelcky, F., Proc. Nat. Acad. Sci., 20, 259 (1934).
}

\title{
SUBSTITUTES FOR PETROL
}

THE need to conserve available supplies of of the amount allotted under the rationing scheme in Great Britain, raises a question as to the applicability of other fuels to the ordinary motorcar or trade vehicle. It has to be borne in mind, however, that at the present time no fuels should be used except for good and sufficient reason, as fuel is a vital supply in the conduct of modern war.
The fact that town's gas was quite largely used in the years 1915-17 as a motor-car fuel suggests that it might once more be brought into service. It will be recalled that the cycle of operations in the petrol engine is essentially the same as that employed in the gas engine. In the former, the liquid fuel is atomized, metered and mixed with air by means of the carburetter, which also provides the means of controlling its flow. When gas 
is to be used, a diffuser of much simpler con. struction than the modern petrol carburetter can be employed, the mixture of fuel and air being effected by a gas supply pipe of about three eighths the diameter of the inlet pipe. On the gas supply pipe a regulating valve is necessary; this might be of the needle type and it might be arranged to operate in conjunction with the accelerator pedal. A main gas tap is obviously needed, because when the engine is shut down the gas must be turned off. For perfect combustion about 6 volumes of air must be supplied; but for economical results 8-10 volumes are better. The power obtainable will be somewhat less than from petrol, but there should be some saving in cost per mile.

For the storage of gas, large and cumbersome gas-bags were used, the obvious place for which is on the roof. As an alternative, steel cylinders containing high-pressure gas can be carried, but as these would generally have to be given some of the interior space of the car, their presence would doubtless be found less acceptable than the swaying bulk of the gas-bag overhead.

The use of gas is in the nature of a reversion to an older system; but it cannot be said that the newer systems offer any possibility of ready adaptation to existing cars. The heavy oils used in Diesel or semi-Diesel engines require a quite different cycle of operations and therefore an essentially different engine. The direct use of powdered fuel is making headway; but it would necessitate an engine approximating to the heavyoil type. A further possibility is that of generating coal gas for the use of the engine; but this would involve the provision of extra plant as well as the adoption of a new technique by the driver.

The most immediately hopeful change-over will, therefore, be to the gas-bag, out of place as it may appear on a smart car. Some gas companies are understood to be planning the supply of gas in bags both at their works and through the garages and service stations, and it may be anticipated that they will arrange also for the provision of gas-carburetters or diffusers so that an easy changeover may be effected. The subject has received little attention in the text-books and reliable data are difficult to obtain. In "Carburetters and Fuel Systems" by A. W. Judge (third edition), illustrations of several carburetters will be found and quite a lot of practical information that would be most useful to anyone considering making this change.

\section{OBIT UARY}

\section{Mr. Sydney Evershed}

$\mathrm{M}^{\mathrm{B}}$ R. SYDNEY EVERSHED died on September 18, at the age of eighty-two years, at his home in Ealing. He had been associated with the manufacture of electrical testing instruments for fiftythree years. As an inventor his influence can be traced in the development of many electrical instruments in many countries in the world. He was the son of John Evershed of Gomshall, Surrey, and his younger brother John, the well-known astronomer, a fellow of the Royal Society, now resides at Ewhurst, Surrey.

At a meeting of the Institution of Electrical Engineers held in 1922 to commemorate the first ordinary meeting of the Society which was held fifty years previously, Mr. Evershed gave some interesting reminiscences of his own early life. In 1876, the year of the Centennial Exhibition in Philadelphia, he read in the newspaper of someone having listened to Hamlet's soliloquy being spoken by Dr. Graham Bell's telephone, and was lost in amazement, as at that time it was commonly believed that this would have required thousands of musical reeds. A year later he saw a drawing of a telephone and was surprised to find how simple its mechanism was. With the help of two baking-powder boxes in which he cut holes in the lids with his knife, a small bar magnet and some silk-covered wire wound round one pole, and a disk of thin sheet iron, which he cut with scissors, near the other, and using a long piece of telegraph wire to form the connecting line between the two crude telephones, he found to his amazement that they worked admirably, the speech transmitted being practically perfect.

He remembered in his own early life in Surrey the days when you saw only one arc lamp at a time, and what a great blaze of light it made to people accus. tomed to the dingy gas lights in the streets! In these days a strenuous research was being made 'to subdivide the electric light'. There was great excitement amongst the physicists when a cablegram came from America saying that Edison had succeeded in dividing it by means of a platinum incandescent lamp. Contemporaneously with this feverish research, Joseph Swan was working in England on his carbon vacuum lamp.

Evershed was then living a few miles from the town of Godalming in Surrey. Strange to say, this quiet Surrey village was the first town in England to light its streets by electricity. A dynamo was installed in Pullman's leather mills, not far from the town, on the river Wey, where water-power was available. The electric lighting of Godalming began on December 15, 1881. He remembered, on a later date, seeing the leather mills lit up with what he was told were Swan lamps. A leather worker with whom 